\title{
Increased risk of breast cancer in neurofibromatosis type I: current insights
}

\author{
This article was published in the following Dove Press journal: \\ Breast Cancer - Targets and Therapy \\ 2I August 2017 \\ Number of times this article has been viewed
}

\author{
Sacha J Howell' \\ Kimberley Hockenhull' \\ Zena Salih' \\ D Gareth Evans ${ }^{2,3}$ \\ 'Department of Medical Oncology, \\ The Christie NHS Foundation Trust, \\ ${ }^{2}$ Division of Evolution and Genomic \\ Sciences, School of Biological \\ Sciences, Faculty of Biology, Medicine \\ and Health, University of Manchester, \\ Manchester Academic Health \\ Science Centre, ${ }^{3}$ Manchester Centre \\ for Genomic Medicine, Central \\ Manchester University Hospitals NHS \\ Foundation Trust, Manchester, UK
}

\begin{abstract}
Neurofibromatosis type 1 (NF1) is an autosomal dominant condition caused by mutation/deletion of the NF1 gene. The gene product, neurofibromin, is a tumor suppressor which represses the activity of the Ras oncogene. Central nervous system (CNS) tumors have long been associated with NF1, but their association with several other malignancies has been demonstrated. In this review, we summarize the epidemiological data that irrefutably support a link between NF1 and an increased risk of early-onset breast cancer, to levels at which annual mammography is currently recommended in national high-risk screening programs. We discuss the reasons for the observed adverse breast cancer prognosis in NF1 cases, including late presentation and more aggressive tumor subtypes, and recommend that a collaborative breast screening study be initiated to better serve this currently underserved population of women.
\end{abstract}

Keywords: breast cancer, neurofibromatosis type 1, NF1

\section{Introduction}

Neurofibromatosis type 1 (NF1) is an autosomal dominant condition with a birth incidence of 1 in 1,900-3,000 and prevalence of approximately 1 in 4,000. ${ }^{1,2} \mathrm{NF} 1$ not only demonstrates complete penetrance but also significant variability in clinical phenotype due to differences in the site and type of genetic defect in the NFI gene and additional genetic and environmental factors that are not well understood. ${ }^{3}$ Clinical features in individuals can be difficult to predict, even within families, which makes genetic counseling imprecise. Approximately 50\% of cases are familial, but the remaining $50 \%$ are sporadic and emerge due to de novo defects in the $N F 1$ gene. $^{4}$

The National Institutes of Health diagnostic criteria for NF1 require two or more of the following; café-au-lait spots, neurofibromas ( 2 or more), skinfold freckling, Lisch nodules, optic glioma, osseous lesions, or a family history of the condition in a firstdegree relative. Genetic testing of the NF1 gene has also allowed molecular diagnosis when the syndrome is suspected and has high sensitivity of approximately $96 \%$ in both de novo and inherited NF1. ${ }^{5}$ Clinical features of NF1 usually become apparent in the first few years of life, with most fulfilling National Institutes of Health criteria between ages 6 and 7. Almost all patients with NF1 develop cutaneous neurofibromas between adolescence and later adulthood, which often cause cosmetic concerns and itching but do not transform into malignant tumors. However, $30 \%-50 \%$ of patients with NF1 also have plexiform neurofibromas, which have the potential to become malignant (malignant peripheral nerve sheath tumors, MPNST). ${ }^{6}$ This link between NF1 and malignancy is well established. The most commonly associated types of cancer are MPNST and intracranial
Correspondence: D Gareth Evans Medicine, Central Manchester University Hospitals NHS Foundation Trust, Manchester, MI3 9WL, UK

Email gareth.evans@cmft.nhs.uk 
gliomas. CNS tumors usually present earlier in childhood and mostly comprise low-grade pilocytic astrocytomas of the optic radiations or brainstem; however, more infrequently, brainstem gliomas occur and are of higher grade. In addition to these tumors, patients with NF1 are at a higher risk of developing gastrointestinal stromal tumors (GIST), rhabdomyosarcomas, and phaeochromocytoma. ${ }^{7}$ Their association with an increased incidence of breast cancer (BC) has been debated for many years, but more conclusive data have now confirmed this link.

The NF1 gene, on chromosome 17, was first characterized in 1990. The gene product is neurofibromin - a large multifunctional protein which acts as a tumor suppressor through induction of RAS GTPase activity - converting active RAS-GTP to inactive RAS-GDP. In addition, neurofibromin1-induced suppression of PI3K/Akt and cAMP signaling has been described. ${ }^{8-10}$ Potential NF1 driver mutations/deletions have been identified in only $3 \%$ of breast tumors. ${ }^{11}$ However, two independent analyses of The Genome Cancer Atlas (TGCA) data have recently demonstrated loss of heterozygosity of NF1 in $28 \%-31 \%$ of cases with unselected invasive $\mathrm{BC}$, increasing to $40 \%-62 \%$ of triple-negative [tumors lacking expression of estrogen, progesterone, and human epidermal growth factor receptors (Her2)] and Her2-enriched subtypes. ${ }^{12,13}$ Reduction in mRNA levels was confirmed, suggesting haplo-insufficiency in NF1 may play an important role in $\mathrm{BC}$ predisposition in NF1 mutation carriers. ${ }^{13}$

Several high-risk BC predisposition genes including BRCA1 and BRCA2 and TP53 have been identified. Germline mutations in these genes often result in BC of adverse pathology in younger women. Lifetime BC risks of $60 \%-85 \%$ have led to high-intensity screening protocols in many countries. For example, in the UK, National Institute For Health and Clinical Excellence (NICE) guidelines suggest offering annual MRIs to BRCA1/2 mutation carriers of ages 30-50 and TP53 carriers from age $20 .{ }^{14}$ In the USA, the National Comprehensive Cancer Network (NCCN) recommends annual breast MRI from age $25 .{ }^{15}$ However, mutations in these genes account for only $\sim 3 \%$ of BC incidence overall. Several additional moderate risk genes have been identified, including $A T M$, BARD1, CHEK2, and PALB2, although appropriate protocols for screening are yet to be defined. It is noteworthy that women at moderate risk of BC due to family history and hormonal risk factors are offered annual mammography between ages 40 and 50 in the UK for a lifetime risk of $17 \%-30 \%$. In this article, we discuss the clinical evidence of increased $\mathrm{BC}$ risk in women with neurofibromatosis and the potential need for a high-risk screening protocol.

\section{Method}

A search of PubMed with neurofibromatosis type 1/neurofibromatosis 1 and breast cancer in the search field in the title was undertaken. Of these, some were case reports of between one and three cases. The others were larger studies of mortality data or genetic profiling of patients with NF1. Key citations from these publications were also crosschecked. Furthermore, UpToDate was reviewed for any further publications, which are summarized in the results.

\section{Results}

We identified 32 published epidemiological studies that investigated a potential association of NF1 diagnosis with invasive cancer risk and, of these, nine examined $\mathrm{BC}$ risks specifically. Most studies were of a similar design, comparing $\mathrm{BC}$ rates \pm mortality, in populations known to have NF1 by clinical and/or genetic criteria, with the background population rates matched for epoch, age, sex, and location. The first epidemiological paper to report the association of NF1 and cancer was in a Danish cohort of 212 NF1 probands identified through hospital admission records. ${ }^{16}$ Data presented included information from 42 years of follow-up in what was presumably a severely affected cohort. Overall, a 4-fold increase in malignant neoplasms or benign CNS tumors was seen in patients with NF1 compared to the general population. Affected female relatives of the probands also showed a 1.9fold (1.1 - to 3.1-fold) increase in risk of predominantly nervous system neoplasms, but BC cases were not documented. In 1995, Zöller et al published a 12-year follow-up study of 70 patients with NF1 in Gothenburg, Sweden. In their mortality analysis, observed deaths $(n=22)$ significantly exceeded those expected $(\mathrm{n}=5 ; p<0.001)$, and $55 \%(12 / 22)$ were due to malignancies, although there was no clear evidence of an excess of BCs. ${ }^{17}$ In an American study of death certification, 3,770 presumed NF1-related deaths were identified from a population of $>32$ million. ${ }^{18}$ The proportional mortality rate (PMR) for malignant neoplasms was significantly elevated in NF1 at 1.21 (1.14-1.28). The predominant neoplasms recorded were those of connective tissues and the brain, with no increase in the recording of breast tumors [PMR 0.66 $(0.52-0.82)]$. However, this study highlights a major issue with death certification studies. In a smaller study of our own, only $36 \%$ of patients with NF1 for whom we were able to obtain details of death certification had NF1 as a contributing cause on the death certificate as did only $1 / 9$ who died from BC. ${ }^{19}$ It is highly likely, therefore, that the association between $\mathrm{BC}$ and NF1 is significantly underestimated. 
More recently, Walker et al designed a prospective study looking at cancer incidence in patients with NF1 using data from the UK NF 1 registry and UK Office of National Statistics. ${ }^{20}$ In 2006, they reported data in 448 NF1 cases, followed for a median of 13.6 years and totaling 5,705 patient years of follow-up. Notably, only the first malignancy in each patient was considered. The standardized incidence ratio (SIR) for all cancer was $2.7(1.9-3.7 ; p<0.0001)$ for the whole population and $3.07(1.9-4.6 ; p<0.0001)$ for the 227 females in the study. The SIR for all female BC was $1.87(0.61-4.37 ; p=0.26)$ but rose to $4.02(1.09-10.3 ; p=0.037)$ for women younger than 50 - the first demonstration of an age-specific increase in BC risk in NF 1 carriers..$^{20}$ Two studies from the North West Regional Genetics Service NF1 Register in the UK have documented an increased $\mathrm{BC}$ incidence ${ }^{21}$ and mortality ${ }^{19}$ in their NF1 cohort. Of 304 female NF1 patients $\geq 20$ years old with a median follow-up of 17.8 years and overall follow-up of 5,411 person years, 14 were diagnosed with $\mathrm{BC}-$ giving an SIR of 3.5 [95\% confidence interval (CI) 1.9-5.9]. In women younger than 50 , this increased to an SIR of 4.9 (95\% CI 2.4-8.8). In terms of mortality, the PMR for BC in the second study was $3.5(95 \% \mathrm{CI}, 1.3-7.7) .{ }^{19}$

As doubt persisted with regard to the link between NF1 and $\mathrm{BC}$, four further epidemiological studies were conducted and reported during 2012-2016. Two US studies from Detroit $^{22}$ and Baltimore ${ }^{6}$ were published in 2012. Wang et $\mathrm{al}^{22}$ studied 76 female NF1 cases $>20$ years and compared BC incidence with the local 4 million participant contribution to the Surveillance Epidemiology and End Results (SEER) registry. Nine cases of BC were identified (two of these nine women had bilateral disease), giving an overall SIR of 5.2 (2.4-9.8). Again, this was predominant in those younger than 50 who had an SIR of 8.8 (3.2-19.2). Of additional interest, the cohort was racially mixed and crude incidence figures appeared similar between African-American women [3 of 22 (13.6\%)] and those described as Caucasian [ 6 of 48 (12.5\%)]. Madanikia et al identified 126 female NF1 cases younger than 20 of whom $28 \%$ were described as Black. Using appropriate time period and race adjustment and comparing $\mathrm{BC}$ incidence with SEER data, the SIR was 2.30 for NF1 cases overall $(p=0.087,95 \%$ CI $0.73-5.55)$ and was highly statistically significant in women younger than 50 (SIR 4.41; $p=0.0049,95 \%$ CI $1.12-12.00$ ). ${ }^{6}$ It is worth noting that these data are based on only four $\mathrm{BC}$ cases, two of whom were diagnosed with $\mathrm{BC}$ prior to intake onto the local NF1 database. The two cases diagnosed after intake had ductal carcinoma in situ (DCIS) and, thus, there is unlikely to be any effect on mortality.

In 2015, Seminog and Goldacre used the Hospital Episode Statistic (HES) dataset, which includes all hospital day cases and inpatient admissions in National Health Service (NHS) hospitals in England to identify cases of NF1 and age-matched controls between 1999 and 2011.23 During this time, 3,672 women were hospitalized with NF1 and, of these, 58 developed $\mathrm{BC}$. The relative risks (RRs) for $\mathrm{BC}$ in 10-year age cohorts in NF1 vs controls were: $30-39$ [RR 6.5 (2.6-13.5)], 40-49 [RR 4.4 (2.5-7.0)], 50-59 [RR 2.6 (1.5-4.2)], 60-69 [RR 1.9 (1.0-3.3)], and 70-79 [RR 0.8 $(0.2-2.2)]$. The authors noted that the estimated absolute risk per year in women aged 30-39 with NF1 ( 1 in 359) was similar to the control population aged 50-59 (1 in 363) - a cohort that is offered regular mammographic screening in most developed health systems.

Two key papers from Finland have recently been published describing SIRs, standardized mortality rates (SMRs), and features of BCs diagnosed in NF1 cases. ${ }^{7,24}$ Data for these papers were taken from all secondary and tertiary medical centers covering the whole of the 5.4 million Finnish population from 1987 to 2011. In total, 737 female NF1 cases were identified and verified according to NIH criteria. Carcinoma-specific survival of patients with NF1 was also compared with that of matched controls from the Finnish Cancer Registry. Overall, the lifetime risk of any cancer was $59.6 \%$ in NF1 cases, compared with $30.8 \%$ in the general Finnish population. The SIR for BC was 3.04 (2.06-4.31, $p<0.001)$ overall and $11.1(95 \%$ CI $5.56-19.5 ; p<0.001)$ in women younger than 40 . Cancer mortality was increased in female NF1 cases overall [SMR $7.23(5.58-9.19 ; p<0.001)$ ] and from BC specifically [SMR $5.20(2.38-9.88 ; p<0.001)]$.

In their follow-up paper, Uusitalo et $\mathrm{al}^{24}$ examined the characteristics of the BCs diagnosed in NF1 cases and controls. Twenty-six of $32 \mathrm{BC}$ samples were retrieved for central analysis and compared with five controls per NF1 sample from a Finnish biobank. Controls had been previously analyzed in the same laboratory using similar methods. Staging information and results of immunohistochemical analysis for estrogen receptor (ER), progesterone receptor (PR), Her2, Ki67, and CK5/6 were obtained and compared with controls. NF1-associated BCs were more often ER negative (53.8\% vs $20.9 \%, p=0.001)$, PR negative $(65.4 \%$ vs $21.7 \%$, $p<0.001$ ), and HER 2 positive ( $30.8 \%$ vs $9.6 \%, p=0.006)$ - all factors associated with adverse prognosis. The NF1 tumors were also larger $(p=0.019)$ and of higher grade $(p=0.050)$. An overall survival analysis was performed against controls matched for age and ER status, demonstrating inferior 5-year survival in those with NF1 [68.1\% (95\% CI 52.0-89.1\%) vs $82.0 \%$ (95\% CI $75.5-88.9 \%) ; p=0.053]$. The hazard ratio for death was 2.3 (95\% CI 0.99-5.6). The group went on to analyze TCGA data and, as with Suárez-Cabrera et $\mathrm{al}^{12}$ and 
Wallace et $\mathrm{al}^{13}$ demonstrated NF1 mutation or deletion in $33 \%$ of BCs, with a significant enrichment in ER-negative and HER2-positive subtypes.

\section{Discussion}

The increased risk of BC in NF1 has now been reported by several groups. The BC risk in those younger than 50 was significantly elevated in all recent studies, with SIRs between 4.0 and 11.1 (Table 1). ${ }^{6,7,20-24}$ Although most of these data are from retrospective studies, the prospective cohort study of Walker et al is consistent with the retrospective data. ${ }^{20}$ Furthermore, the inclusion of only first cancer diagnoses in this study may underestimate the risk of BC by excluding women with multiple malignancies. As an example, in the study of Wang et al, ${ }^{22}$ of the 13 women with a diagnosis of invasive or in situ BC, three had another primary tumor that would have precluded BC registration in Walker et al's study if occurring prior to the $\mathrm{BC}$ diagnosis. ${ }^{20}$ The only study to show a possible reduction in the incidence of $\mathrm{BC}$ was the death certification study of Rasmussen et al (SIR 0.66). ${ }^{18}$ We have previously highlighted the lack of reliability of death certification data in identifying NF1 cases, particularly those who have died from causes not readily identified as being secondary to NF1 itself. ${ }^{19}$ Despite these potential issues with study design, the data are remarkably consistent and provide irrefutable data linking NF1 with an increased risk of BC which is diagnosed at younger age than in the general population and with poorer outcomes. The poorer outcome is likely due to the increase in triple-negative (ER, PR, and HER2) and HER2positive tumor subtypes, which are associated with inferior 5-year survival compared with ER-positive HER2-negative cancers. ${ }^{29}$ In addition, Uusitalo et al confirmed a significant increase in T stage with NF1 when compared with the general population. ${ }^{24}$ Overall survival in their cohort was significantly worse even after matching tumors in NF1 and controls for
ER status. However, BC-specific survival would have been a more meaningful endpoint in this situation in view of the widely reported inferior survival in NF1, which is driven by both neoplastic and cardiovascular risks.

Notwithstanding this issue, the PMR reported in the study from Manchester confirms the adverse outcomes with NF1associated BC, and a combination of aggressive tumor phenotypes and later presentation are the most likely causes. ${ }^{19}$ For women who are used to having benign breast lumps, it is perhaps understandable that they may delay initial presentation when another lump arises. Even at presentation, there can be difficulties in diagnosis as mammograms can be more challenging to interpret in NF1 cases. ${ }^{25,26}$ Although neurofibromas are generally well circumscribed and oval/round on mammography, they may obscure neoplastic lesions. ${ }^{26} \mathrm{MRI}$ may be a more appropriate imaging technique, although its reduced specificity versus mammography leads to increased recall rates in the general screening population and this could theoretically be even higher in NF1 carriers. ${ }^{27}$ Only one case report has been published documenting the MRI appearance of NF1 breast neurofibromas, and a prospective study in this group is clearly required. ${ }^{25}$

If we consider the indication for a breast screening program in NF1, we must relate this to high-risk screening programs that are already established. In the UK, the updated high-risk screening protocol defines population risk as $<3 \%$ risk of $\mathrm{BC}$ aged $40-50$, moderate risk as $3-8 \%$, and high risk as $>8 \%{ }^{14}$ For women at moderate risk, annual mammography is recommended from 40 to 49 and, for those at high risk, mammography is offered from 40 to 59 and considered from 30 to 39 . In addition, those at very high risk due to $B R C A 1 / 2$ or TP53 mutation are offered annual MRI. In Table 1, we have calculated the 10-year BC risks from the six most recent epidemiological studies in NF1 patients. All studies would fulfill the criteria for at least moderate risk screening with annual surveillance at 40-50, with the Finish study suggesting that screening should

Table I 10 year breast cancer risks aged 30 and 40 years in NFI patients from population based and cohort studies with Standard Incidence ratios

\begin{tabular}{|c|c|c|c|c|c|}
\hline Ref & SIR $<50(95 \% \mathrm{Cl})$ & $\begin{array}{l}10 \text {-year BC risk } \\
30 \text { years general } \\
\text { population UK }\end{array}$ & $\begin{array}{l}\text { Estimated } \\
\text { I0-year BC risk } \\
30 \text { years NFI UK }\end{array}$ & $\begin{array}{l}10 \text {-year } B C \\
\text { risk aged } \\
40 \text { years general } \\
\text { population UK }\end{array}$ & $\begin{array}{l}\text { Estimated I0-year } \\
\text { BC risk aged } 40 \\
\text { years in NFI UK }\end{array}$ \\
\hline Walker et $\mathrm{al}^{20}$ & $4.02(1.09-10.3)$ & $0.5 \%$ & $2 \%$ & $1.6 \%$ & $6.5 \%$ \\
\hline Sharif et $\mathrm{al}^{21}$ & $4.9(2.4$ to 8.8$)$ & $0.5 \%$ & $2.5 \%$ & $1.6 \%$ & $7.8 \%$ \\
\hline Wang et $\mathrm{a}^{22}$ & $8.8(3.2-19.2)$ & $0.5 \%$ & $4.4 \%$ & $1.6 \%$ & $14 \%$ \\
\hline Madanikia et $a^{6}$ & $4.4 \mathrm{I}(1.12$ to 12.00$)$ & $0.5 \%$ & $2.2 \%$ & $1.6 \%$ & $7 \%$ \\
\hline Seminog and Goldacre ${ }^{23}$ & $\begin{array}{l}\text { 30-39 RR } 6.5 \text { (2.6-13.5), } \\
40-49 \text { RR } 4.4 \text { (2.5-7.0), }\end{array}$ & $0.5 \%$ & $3.2 \%$ & $1.6 \%$ & $7 \%$ \\
\hline Uusitalo et $\mathrm{al}^{7,24}$ & $\begin{array}{l}<40 \text { II.I }(5.6-19.5) \\
40-492.6(0.95-5.65)\end{array}$ & $0.5 \%$ & $5.5 \%$ & $1.6 \%$ & $4.2 \%$ \\
\hline
\end{tabular}

Abbreviations: BC, breast cancer; NFI, neurofibromatosis type I; RR, relative risk; SIR, standardized incidence ratio. 
start earlier than this. ${ }^{7,24}$ Notably, the most recent version of the NCCN guidelines (Genetic/Familial High-Risk Assessment: Breast and Ovarian, Version 2.2017) advise annual mammogram starting at age 30 and consideration of breast MRI with contrast from ages 30 to 50 in the NF1 population. ${ }^{30}$ Ovarian cancer risk was noted not to be increased with $N F 1$ mutation. ${ }^{30}$ The safety of mammography in the NF1 population is not known. Although the radiation exposure is low with mammography, this group have been shown to develop second tumors in response to therapeutic ionizing radiation. ${ }^{28}$ In view of this and the observations that BCs in the NF1 population occur in younger women, where mammography is less sensitive and are more frequently of adverse prognostic subtypes, we believe it is time to institute a collaborative multinational MRI-based breast screening study to quantify the potential benefits and harms for patients with NF1.

\section{Disclosure}

The authors report no conflicts of interest in this work.

\section{References}

1. Evans DG, Howard E, Giblin C, et al. Birth incidence and prevalence of tumor-prone syndromes: estimates from a UK family genetic register service. Am J Med Genet A. 2010;152A(2):327-332.

2. Uusitalo E, Leppävirta J, Koffert A, et al. Incidence and mortality of neurofibromatosis: a total population study in Finland. J Invest Dermatol. 2015;135(3):904-906.

3. Easton DF, Ponder MA, Huson SM, Ponder BA. An analysis of variation in expression of neurofibromatosis (NF) type 1 (NF1): evidence for modifying genes. Am J Hum Genet. 1993;53(2):305-313.

4. Abramowicz A, Gos M. Neurofibromin in neurofibromatosis type $1-$ mutations in NF1 gene as a cause of disease. Dev Period Med. 2014;18(3): 297-306.

5. Evans DG, Bowers N, Burkitt-Wright E, et al; Northern UK NF1 Research Network. Comprehensive RNA analysis of the NF1 gene in classically affected NF1 meeting NIH criteria has high sensitivity and mutation negative testing is reassuring in isolated cases with pigmentary features only. EBioMedicine. 2016;7:212-220.

6. Madanikia SA, Bergner A, Ye X, Blakeley JO. Increased risk of BC in women with NF1. Am J Med Genet A. 2012;158A(12):3056-3060.

7. Uusitalo E, Rantanen M, Kallionpää RA, et al. Distinctive cancer associations in patients with neurofibromatosis type 1. J Clin Oncol. 2016;34(17):1978-1986.

8. Johannessen CM, Reczek EE, James MF, Brems H, Legius E, Cichowski $\mathrm{K}$. The NF1 tumor suppressor critically regulates TSC2 and mTOR. Proc Natl Acad Sci U S A. 2005;102(24):8573-8578.

9. Hegedus B, Dasgupta B, Shin JE, et al. Neurofibromatosis-1 regulates neuronal and glial cell differentiation from neuroglial progenitors in vivo by both cAMP- and Ras-dependent mechanisms. Cell Stem Cell. 2007;1(4):443-457.

10. Tong J, Hannan F, Zhu Y, Bernards A, Zhong Y. Neurofibromin regulates G protein-stimulated adenylyl cyclase activity. Nat Neurosci. 2002;5(2):95-96.
11. Cancer Genome Atlas Network. Comprehensive molecular portraits of human breast tumours. Nature. 2012;490(7418):61-70.

12. Suárez-Cabrera C, Quintana RM, Bravo A, et al. A transposon-based analysis reveals RASA1 is involved in triple-negative breast cancer. Cancer Res. 2017;77(6):1357-1368.

13. Wallace MD, Pfefferle AD, Shen L, et al. Comparative oncogenomics implicates the neurofibromin 1 gene (NF1) as a breast cancer driver. Genetics. 2012;192(2):385-396.

14. National Institute for Health and Care Excellence (NICE) NICE.org. uk. Available from: https:/www.nice.org.uk/guidance/cg164/chapter/ recommendations\#summary-of-recommendations-on-surveillance-forwomen-with-no-personal-history-of-breast-cancer. Accessed March 5, 2017.

15. National Comprehensive Cancer Network (NCCN) website. Available from: https://www.nccn.org/professionals/physician_gls/f_guidelines. asp\#genetics_screening. Accessed March 5, 2017.

16. Sørensen SA, Mulvihill JJ, Nielsen A. Long-term follow-up of von Recklinghausen neurofibromatosis: survival and malignant neoplasms. N Engl J Med. 1986;314(16):1010-1015.

17. Zöller M, Rembeck B, Akesson HO, Angervall L. Life expectancy, mortality and prognostic factors in neurofibromatosis type 1. A twelveyear follow-up of an epidemiological study in Goteborg, Sweden. Acta Derm Venereol. 1995;75(2):136-140.

18. Rasmussen SA, Yang Q, Friedman JM. Mortality in neurofibromatosis 1: an analysis using U.S. death certificates. Am J Hum Genet. 2001;68(5): 1110-1118.

20. Walker L, Tompson D, Easton D, et al. A prospective study of neurofibromatosis type 1 cancer incidence in the UK. Br J Cancer. 2006;95(2): 233-238.

21. Sharif S, Moran A, Huson SM, et al. Women with neurofibromatosis 1 are at a moderately increased risk of developing breast cancer and should be considered for early screening. J Med Genet. 2007;44(8):481-484.

22. Wang X, Levin AM, Smolinski SE, Vigneau FD, Levin NK, Tainsky MA Breast cancer and other neoplasms in women with neurofibromatosis type 1: a retrospective review of cases in the Detroit metropolitan area. Am J Med Genet A. 2012;158A(12):3061-3064.

23. Seminog OO, Goldacre MJ. Risk of benign tumours of nervous system, and of malignant neoplasms, in people with neurofibromatosis: population-based record-linkage study. Br J Cancer. 2013;108(1):193-198.

24. Uusitalo E, Kallionpää RA, Kurki S, et al. Breast cancer in neurofibromatosis type 1: overrepresentation of unfavourable prognostic factors. Br J Cancer. 2017;116(2):211-217.

25. Gokalp G, Hakyemez B, Kizilkaya E, Haholu A. Myxoid neurofibromas of the breast: mammographical, sonographical and MRI appearances. Br J Radiol. 2007;80(958):e234-e237.

26. Zhou Y, Pan B, Mao F, et al. A hidden breast lump covered by nipple appendices in a patient with von Recklinghausen disease: a case report and review of the literature. Clin Breast Cancer. 2012;12(1):71-75.

27. Leach MO, Boggis CR, Dixon AK, et al; MARIBS study group. Screening with magnetic resonance imaging and mammography of a UK population at high familial risk of breast cancer: a prospective multicentre cohort study (MARIBS). Lancet. 2005;365(9473):1769-1778.

28. Sharif S, Ferner R, Birch JM. Second primary tumours in neurofibromatosis 1 patients treated for optic glioma: substantial risks after radiotherapy. J Clin Oncol. 2006;24(16):2570-2575.

29. Sorlie T, Tibshirani R, Parker J, et al. Repeated observation of breast tumor subtypes in independent gene expression data sets. Proc Natl Acad Sci U S A. 2003;100(14):8418-8423.

30. Daly MB, Pilarski R, Berry M, et al. NCCN Guidelines Insights: Genetic/Familial High-Risk Assessment: Breast and Ovarian, Version 2.2017. J Natl Compr Canc Netw. 2017;15(1):9-20. 


\section{Publish your work in this journal}

Breast Cancer - Targets and Therapy is an international, peerreviewed open access journal focusing on breast cancer research, identification of therapeutic targets and the optimal use of preventative and integrated treatment interventions to achieve improved outcomes, enhanced survival and quality of life for the cancer patient.
The manuscript management system is completely online and includes a very quick and fair peer-review system, which is all easy to use. Visit http://www.dovepress.com/testimonials.php to read real quotes from published authors.

Submit your manuscript here: https://www.dovepress.com/breast-cancer---targets-and-therapy-journal 